Supporting Information for

\title{
A linear relationship between the charge transfer amount and the level alignment in molecule/two- dimensional adsorption systems
}

\author{
Rui Hou, ${ }^{\dagger, \downarrow}$ Yang Xia, ${ }^{\S, \|}$ and Shenyuan Yang ${ }^{*,+,}$ \\ ${ }^{\dagger}$ State Key Laboratory of Superlattices and Microstructures, Institute of Semiconductors, \\ Chinese Academy of Sciences, Beijing 100083, China \\ ${ }^{\ddagger}$ College of Materials Science and Opto-electronic Technology, University of Chinese \\ Academy of Sciences, Beijing 100049, China \\ ${ }^{\S}$ Institute of Microelectronics of Chinese Academy of Sciences, Beijing 100029, China \\ "University of Chinese Academy of Sciences, Beijing 100049, China
}




\section{Adsorption geometries of molecule/2D systems}

(a) $\mathrm{TTF} / \mathrm{MoS}_{2}$

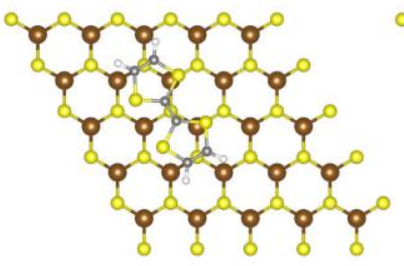

(d)

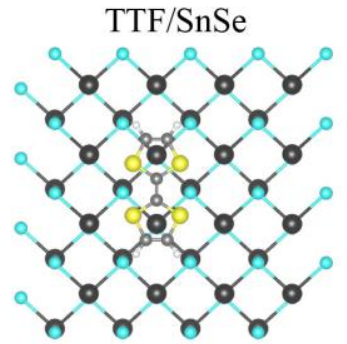

(g)

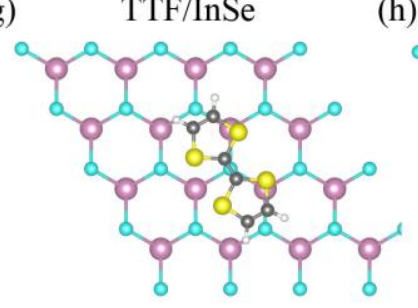

- Mo

$\mathrm{H}$ (b) $\mathrm{TCNQ} / \mathrm{MoS}_{2}$

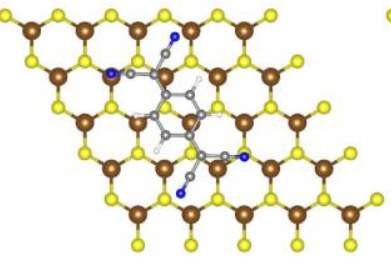

(e)

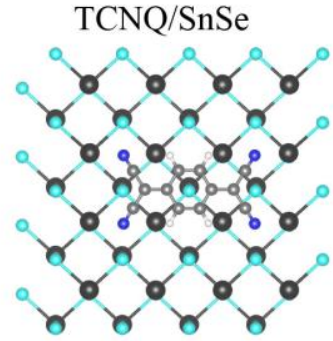

(h)

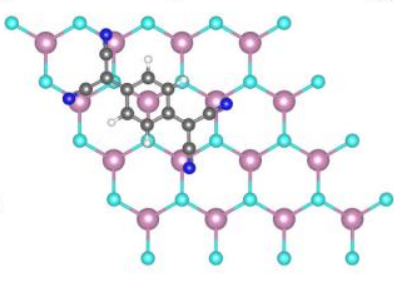

$\circ \mathrm{Se}$

- $\mathrm{Sn}$

$\circ \mathrm{S}$ (c) $\mathrm{TCNE} / \mathrm{MoS}_{2}$

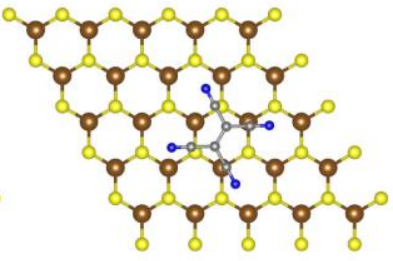

(f)

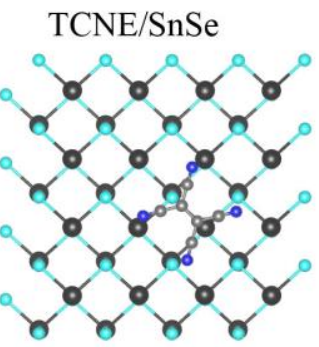

(i) $\quad \mathrm{TCNE} / \mathrm{InSe}$

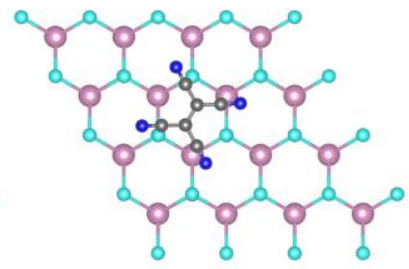

In

- $\mathrm{N}$

Figure S1. The adsorption geometries of (a) TTF/MoS 2 , (b) TCNQ/MoS 2 , (c) TCNE/MoS 2 , (d) TTF/SnSe, (e) TCNQ/SnSe, (f) TCNE/SnSe, (g) TTF/InSe, (h) TCNQ/InSe, and (i) TCNE/InSe.

Figure S1 shows the geometries of other molecule/2D adsorption systems. The three molecules align along the zigzag direction of $\mathrm{MoS}_{2}$. The midpoint of the $\mathrm{C}-\mathrm{C}$ double bond 
of TTF is directly above the $\mathrm{S}$ atom in $\mathrm{MoS}_{2}$ (Figure S1a). The center of the benzene ring of TCNQ is above the midpoint of the Mo-S bond (Figure S1b), while the center of TCNE is above the center of the hexagon of $\mathrm{MoS}_{2}$ (Figure S1c). The adsorption configurations on other TMDs monolayers are similar to those on $\mathrm{MoS}_{2}$. The adsorption geometries of the three molecules on SnSe (Figure S1d,e,f) are similar to those on BP monolayer. The adsorption geometries of TTF and TCNE on InSe and InS (Figures S1g,i) are similar to those on $\mathrm{MoS}_{2}$. TCNQ aligns along the armchair direction of InSe, with the center of the benzene ring above the In atom (Figure S1h). 


\section{Partial charge densities of molecule/2D systems}

(a)

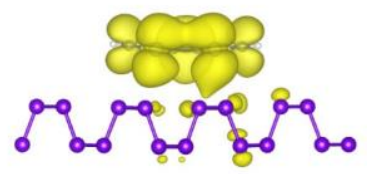

(c)

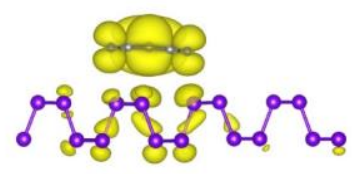

(b)

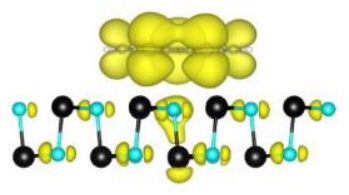

(d)

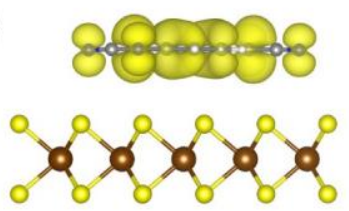

Figure S2. Partial charge densities of (a) the HOMO level of TTF in TTF/BP, (b) the HOMO level of TTF in TTF/SnSe, (c) the LUMO level of TCNQ in TCNQ/BP and (d) the LUMO level of TCNQ in TCNQ/MoS 2 . The isosurface value is $0.002 \mathrm{e} \cdot \AA^{-3}$.

As shown in Figure 2 (c), the energy range of the HOMO level of TTF molecule in $\mathrm{TTF} / \mathrm{BP}$ is between -4.69 and $-4.45 \mathrm{eV}$. The partial charge density of this HOMO level is shown in Figure S2a. As we can see, this HOMO level is distributed on both BP and TTF, indicating a hybridization between the TTF HOMO level and the BP CBM state. This hybridization results in a charge transfer from TTF molecule to the BP monolayer. As shown in Figure S2c, the partial charge density of the LUMO level of TCNQ in TCNQ/BP 
systems also distributed on the molecule and the monolayer, indicating a charge transfer from BP VBM state to the TCNQ LUMO level.

On the other hand, the partial charge density of the LUMO level of TCNQ in $\mathrm{TCNQ} / \mathrm{MoS}_{2}$ is only distributed on the molecule itself, as shown in Figure S2d. Therefore, there is no hybridization between the TCNQ LUMO level and the $\mathrm{MoS}_{2}$ band edge states, resulting in nearly zero charge transfer between the molecule and the monolayer. For TTF/SnSe, the HOMO level of TTF molecule ranges from -4.67 to $-4.37 \mathrm{eV}$ (Figure 2f), and the VBM state of SnSe happens to appear in this energy range. That is why the partial charge density in Figure S2b also shows a small amount of charge distribution on the SnSe layer. In fact, there is no evident hybridization between the TTF LUMO level and the SnSe states. And Bader analysis indeed gives a very small value for the charge transfer. 


\section{Level alignments and charge transfer amounts of other molecule/2D systems}

Table S1. The HOMO and LUMO levels of molecules and the charge transfer amounts $\Delta n$ between these molecules and the four 2D monolayers.

\begin{tabular}{lllllll}
\hline & HOMO & LUMO & \multicolumn{4}{c}{$\Delta n(\mathrm{e} /$ molecule $)$} \\
\cline { 5 - 7 } & $(\mathrm{eV})$ & $(\mathrm{eV})$ & $\mathrm{MoS}_{2}$ & $\mathrm{WS}_{2}$ & $\mathrm{BP}$ & $\mathrm{InSe}$ \\
\hline $\mathrm{O}_{2}$ & -7.37 & -5.15 & $+0.041^{48}$ & +0.026 & $+0.064^{50}$ & $+0.015^{51}$ \\
$\mathrm{~N}_{2}$ & -8.41 & -4.60 & - & - & - & $+0.001^{51}$ \\
$\mathrm{NO}$ & -4.91 & -3.72 & $+0.022^{48}$ & $+0.018^{49}$ & $+0.074^{50}$ & $+0.018^{51}$ \\
$\mathrm{O}_{3}$ & -8.27 & -6.60 & $+0.126^{52}$ & - & - & - \\
$\mathrm{NO}_{2}$ & -6.93 & -5.61 & $+0.02^{53}$ & +0.084 & +0.248 & $+0.072^{51}$ \\
$\mathrm{H}_{2}$ & -10.47 & -1.75 & $+0.008^{48}$ & $+0.002^{49}$ & $-0.013^{55}$ & $-0.146^{54}$ \\
$\mathrm{CO}$ & -9.28 & -2.32 & $+0.020^{48}$ & $+0.022^{49}$ & $-0.007^{50}$ & $+0.006^{51}$ \\
$\mathrm{H}_{2} \mathrm{O}$ & -7.46 & -1.34 & $+0.016^{48}$ & $+0.017^{49}$ & $-0.035^{50}$ & $+0.014^{51}$ \\
$\mathrm{NH}_{3}$ & -6.42 & -1.68 & $-0.02^{53}$ & $-0.061^{49}$ & $-0.050^{50}$ & $-0.025^{51}$ \\
$\mathrm{DMPD}^{5}$ & -4.25 & -1.61 & $-0.13^{31}$ & - & - & - \\
\hline
\end{tabular}

We note that the spin-polarized calculations are necessary for $\mathrm{NO}_{2} / 2 \mathrm{D}$ and $\mathrm{O}_{2} / 2 \mathrm{D}$

systems since these molecules are spin-polarized. The non-spin-polarized calculations would predict inaccurate charge transfer values for these systems due to incorrect positions and/or occupations of the molecular orbitals. The charge transfer amounts $\Delta n$ for $\mathrm{NO}_{2} / \mathrm{BP}$, $\mathrm{NO}_{2} / \mathrm{WS}_{2}$, and $\mathrm{O}_{2} / \mathrm{WS}_{2}$ from our spin-polarized calculations are shown in Table S1, and the other values are taken from previous reports. ${ }^{31,48-55}$ Among all the systems, only three 
systems $\left(\mathrm{NO}_{2} / \mathrm{BP}, \mathrm{O}_{3} / \mathrm{MoS}_{2}\right.$, and $\left.\mathrm{DMPD} / \mathrm{MoS}_{2}\right)$ are in the charge transfer regions. These three systems follow the same linear relationship as shown in Figures 4 b,c. The other systems with either $\Delta E_{\mathrm{CH}}>0$ or $\Delta E_{\mathrm{VL}}<0$ have much smaller charge transfer amounts. 


\section{Adsorption geometries of $\mathrm{Li}$ and $\mathrm{F}$ atoms on $\mathrm{MoS}_{2}$ monolayer}

(a)
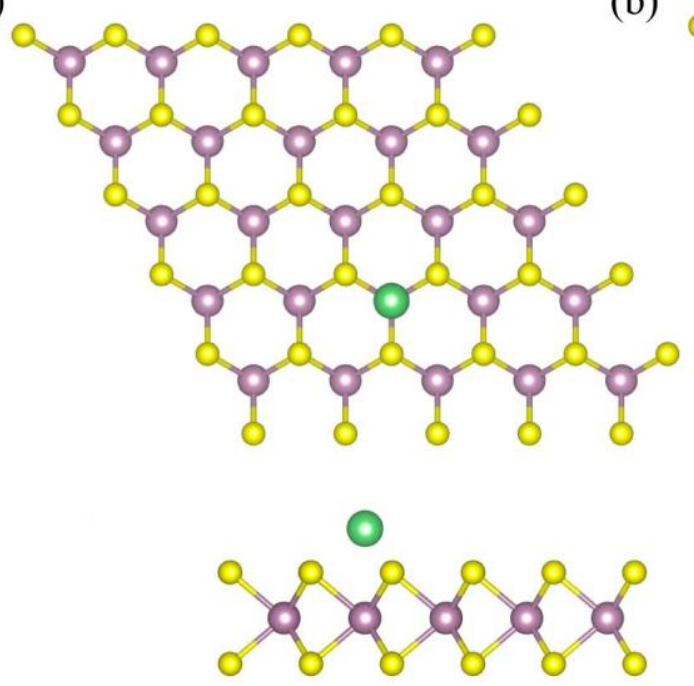

(b)

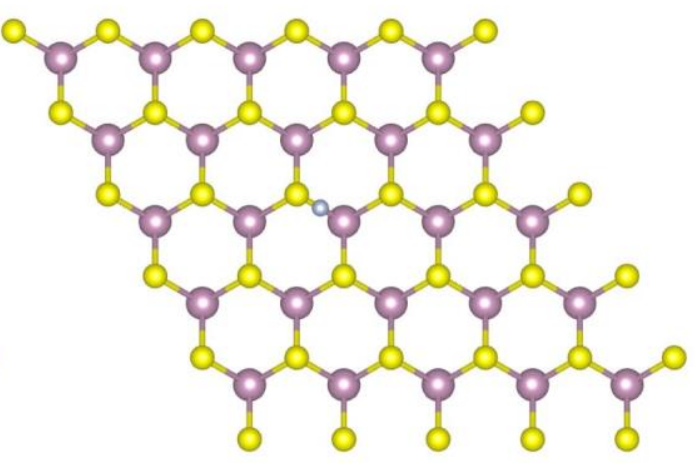

○

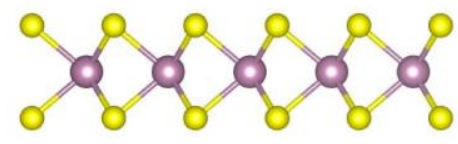

Figure S3. Top and side views of (a) $\mathrm{Li} / \mathrm{MoS}_{2}$ and (b) F/MoS 2 .

As shown in Figure S3, Li atom adsorbs directly above Mo atom and F atom is located above the $\mathrm{Mo}-\mathrm{S}$ bridge on $\mathrm{MoS}_{2}$ monolayer. The distances $h$ between the adsorbed atom and the top surface of $\mathrm{MoS}_{2}$ are 1.49 and $1.81 \AA$ for $\mathrm{Li} / \mathrm{MoS}_{2}$ and $\mathrm{F} / \mathrm{MoS}_{2}$, respectively, in agreement with previous study. ${ }^{\mathrm{a}}$ 


\section{REFERENCES:}

(a) Li, X. D.; Fang, Y. M.; Wu, S. Q.; Zhu, Z. Z. Adsorption of Alkali, Alkaline-Earth, Simple and 3d Transition Metal, and Nonmetal Atoms on Monolayer MoS $2 . A I P A d v$. 2015,

$5,057143$. 